\title{
Pour un classement des crèmes de ramassage
}

\author{
par \\ R. ALIFAX \\ Laboratoire de Recherches de la Chaire des Industries Agricoles \\ et Alimentaires, Institut National Agronomique, \\ 16, rue Claude-Bernard, Paris $5^{\mathrm{e}}$
}

\section{I. - INTRODUCTION}

Dans l'élaboration du beurre, et en dépit de tous les perfectionnements techniques mis en œuvre dans l'industrie, les caractéristiques de la matière première exercent sur le produit fini une importance considérable.

C'est ainsi que nous sommes amenés à distinguer, de ce point de vue, entre crèmes "laitières 》 et crèmes "fermières ».

Quand l'usine beurrière reçoit du lait dont elle sépare la crème, l'opération se fait à un rythme et dans des conditions telles que le produit obtenu, la crème laitière, peut être contrôlé et amélioré assez aisément.

Par contre, dans le cas des crèmes élaborées à la ferme, les conditions d'écrémage, de stockage et de collecte sont parfois peu satisfaisantes, d'où résultent des modifications qui ne sont pas sans exercer une fâcheuse influence sur les caractéristiques du produit fini.

Dans la crème fermière, en particulier, les données ayant trait aux délais d'attente du produit sont extrêmement variables : le beurre est obtenu à partir de crèmes ayant des âges très différents et ayant subi des températures également différentes ; il est considéré généralement comme beaucoup plus sensible que le beurre "laitier " aux altérations de la matière grasse.

Une méthode de classement des crèmes de ramassage serait, pour le beurrier qui traite de telles matières premières, d'un concours précieux.

Un tel classement lui permettrait en effet de traiter séparément les crèmes selon leurs qualités, et d'éviter ainsi des fabrications hétérogènes qui exposeraient inévitablement le produit final aux phénomènes d'altération mentionnés ci-dessus. 
Il nous a donc paru souhaitable d'étudier une technique simple, rapide et d'interprétation facile qui, venant à l'appui des données organoleptiques, contribuerait à résoudre ce problème.

\section{II. - ESSAIS}

Nous nous sommes efforcés de traduire, au cours d'essais successifs, les modifications de nature bactériologique, biochimique ou chimique, qui peuvent se produire dans divers échantillons de crème crue, maintenue à des températures différentes et souillée volontairement ou non. Ces modifications seraient consécutives au développement et au métabolisme de sa flore, et à l'altération de ses constituants.

\section{a) Flore développée}

\section{Ferments lactiques}

Les conditions de travail en France rendent la solution du problème particulièrement délicate. En effet, la plupart des usines travaillent des crèmes acides. Ces crèmes ne sont pas, a priori de mauvaise qualité, surtout si l'acidité résulte du développement de la flore lactique et l'on conçoit aisément qu'un titrage d'acidité, une mesure du $\mathrm{pH}$, une épreuve au bleu de méthylène, ne puissent être d'aucune signification ou d'aucun intérêt Aussi avons-nous orienté nos investigations vers la recherche des techniques ne faisant pas intervenir directement l'acidité.

\section{$2^{\circ}$ Recherche des levures et moisissures}

Avec les ferments lactiques, les levures et les moisissures sont, parmi les agents de contamination, le plus souvent rencontrées dans les crèmes de ramassage. Elles sont en effet responsables des goûts de malt et de moisi et partiellement du goût de fromage. Nous avons cherché à savoir si une détermination rapide et indirecte de la flore fongique pouvait offrir un intérêt dans le cas qui nous préoccupe. Nous avons, pour cela, essayé le test de sédimentation de Wildman [2]. En voici, brièvement résumé, le principe : dans une crème, les moisissures s'agglutinent quand celle-ci est chauffée après addition d'une solution aqueuse de bleu de Méthylène à 0,5 p. 100 contenant du borate de sodium à 20 p. 1000 .

Le dépôt correspondant à $5 \mathrm{ml}$ de crème est récolté et posé sur une plaque. On en évalue approximativement le diamètre qui est proportionnel à la contamination de l'échantillon. L'appréciation des résultats est difficile et délicate ; de plus, la technique est longue, peu pratique. Elle ne peut donc pas être entreprise en série. 


\section{b) Métabolisme de la flore}

Recherche des substances réductrices par l'utilisation des sels du Tryphényltétrazolium

Nous avons entrepris ensuite des essais dans le but de traduire, par une technique chimique appropriée, les modifications survenues dans la crème aux dépens de ses constituants sous l'influence de sa flore totale.

Nous avons tout d'abord pensé à utiliser la propriété que possèdent les sels du Tryphényltétrazolium (chlorure ou bromure) de réagir avec les substances réductrices pour donner du formazan de coloration rouge, en supposant, bien entendu, que ces composés pouvaient résulter du métabolisme du peuplement microbien de la crème analysée.

Ce test ne nous a pas donné les résultats souhaités. La coloration est instable et tous les tubes, dans un très court délai, présentent une teinte uniforme. Une lecture comparative devient impossible.

\section{c) Mise en évidence des indices d'altération des corps gras}

Nous avons cherché à mettre en évidence les modifications produites au niveau de la matière grasse en faisant appel aux indices classiques d'altération des corps gras. Nous avons renoncé aux indices de peroxyde et d'acide thiobarbiturique, d'application trop lente et trop onéreuse. Par contre, nous nous sommes intéressés à la réaction des groupements carbonylés qui seraient éventuellement présents dans les crèmes en voie d'altération.

Pour le déceler nous avons utilisé la réaction de ces composés avec la 2-4 dinitrophénylhydrazine (D.N.P.H.) qui donne des hydrazones pouvant être colorées par la potasse en solution alcoolique.

Le mode opératoire suivant a été adopté :

Dans un tube à essais, on verse $2 \mathrm{ml}$ d'alcool éthylique purifié par reflux pendant $1 \mathrm{~h}$, puis par distillation.

On ajoute ensuite :

- $2 \mathrm{ml}$ de crème,

- $1 \mathrm{ml}$ d'une solution de $2-4$ dinitrophénylhydrazine (D.N.P.H.) à 0,5 p. 1000 dans l'alcool éthylique purifié comme il est indiqué ci-dessus.

Le tube est alors immergé dans un bain-marie maintenu à $60^{\circ} \mathrm{C}$ pendant $30 \mathrm{mn}$. On ajoute ensuite $1 \mathrm{ml}$ d'une solution alcoolique de $\mathrm{KOH}$ à 4 p. 100. Le volume est amené finalement à $10 \mathrm{ml}$ et le tube est laissé à la température de la salle. Après $10 \mathrm{mn}$ de contact, une appréciation de la couleur développée devient déjà possible et peut orienter le praticien.

La sensibilité de l'épreuve peut cependant être augmentée en ajoutant $2 \mathrm{ml}$ d'un mélange volume à volume d'oxyde d'éthyle, d'al- 
cool éthylique et d'alcool isoamylique. On agite, on laisse déposer ou mieux on centrifuge. On compare ensuite les colorations.

\section{III. - ESSAIS EXPERIMENTAUX}

Sur 6 échantillons de crème, d'âge et de fraîcheur différents, nous avons déterminé l'acidité Dornic, procédé aux examens bactériologiques : levures et moisissures, coliformes, caséolytiques, lipolytiques.

Les levures et moisissures ont été dénombrées sur le milieu au lactosérum de fromagerie, gélosé à $\mathrm{pH} 4,5$.

Les coliformes, sur gélose au désoxycholate.

Les caséolytiques, sur le milieu au caséinate de calcium.

Les lipolytiques, sur le milieu que nous avons nous-mêmes décrit [1].

Nous avons ensuite sur ces échantillons appliqué le test au D.N.P.H. décrit ci-dessus. A la suite de cette dernière épreuve, nous avons photographié les tubes dans lesquels les réactions au D.N.P.H. avaient été réalisées. Nous avons par la suite retenu trois types de coloration, susceptibles d'interpréter les qualités des différents échantillons de crème soumis à nos essais.

a) Résultats de la photographie. Le cliché ci-après montre les trois types de coloration (I), (II) et (III).

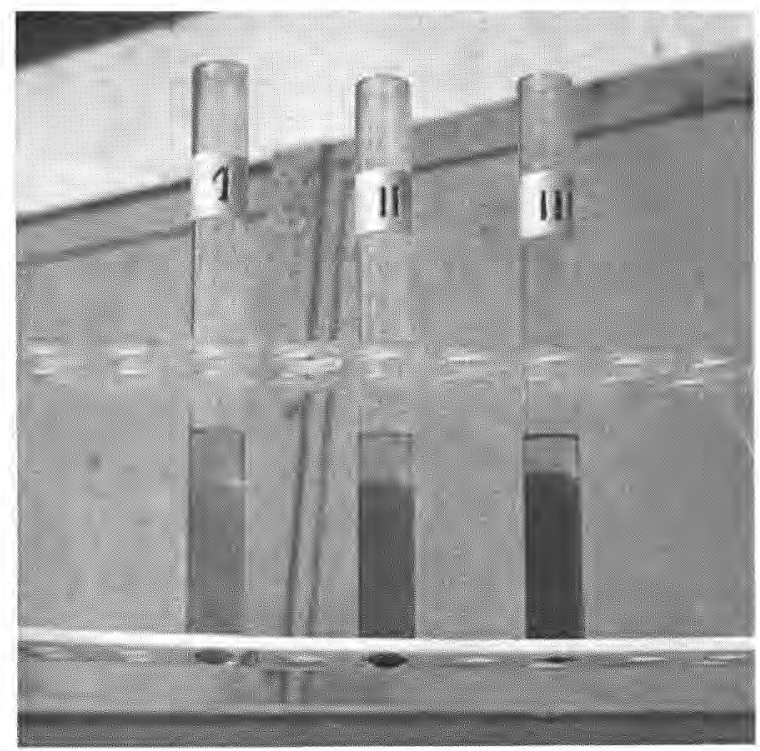


Pour simplifier, nous croyons qu'il serait beaucoup plus commode de les limiter à deux groupes (I) et (II - III).

b) Résultats généraux

\begin{tabular}{c|c|c|c|c|c|c|c}
\hline & \multirow{2}{*}{ Echantillons } & $\begin{array}{c}\text { Acidités } \\
\text { en } \\
{ }^{\circ} \mathrm{D}\end{array}$ & \multicolumn{3}{|c|}{$\begin{array}{c}\text { Analyses bactériologiques } \\
\text { germes par ml }\end{array}$} & \multicolumn{2}{c}{$\begin{array}{c}\text { Classement par le } \\
\text { test au D.N.P.H. }\end{array}$} \\
\cline { 3 - 7 } & $\begin{array}{c}\text { Levures } \\
\text { moisis. }\end{array}$ & $\begin{array}{c}\text { Coli- } \\
\text { formes }\end{array}$ & $\begin{array}{c}\text { Caséoly- } \\
\text { tiques }\end{array}$ & $\begin{array}{c}\text { Lipoly- } \\
\text { tiques }\end{array}$ & $\begin{array}{c}\text { Colora- } \\
\text { tions }\end{array}$ & Qualités \\
\hline & & & & & & & \\
\hline 1 & 25 & $17,10^{3}$ & $24,10^{3}$ & $40,10^{3}$ & 0 & I & $1^{\text {re }}$ \\
2 & 40 & $29,10^{5}$ & $39,10^{4}$ & $6,10^{4}$ & 0 & II-III & $2^{\text {me }}$ \\
3 & 65 & $14,10^{5}$ & $36,10^{5}$ & $11,10^{5}$ & 0 & II-III & $2^{\text {me }}$ \\
4 & 52 & $30,10^{6}$ & $21,10^{7}$ & $10,10^{4}$ & 6 & II-III & $2^{\text {me }}$ \\
5 & 59 & $52,10^{4}$ & $48,10^{5}$ & $13,10^{3}$ & 0 & I & $1^{\text {re }}$ \\
6 & 95 & $74,10^{9}$ & $52,10^{8}$ & $54,10^{4}$ & 0 & II-III & $2^{\text {me }}$ \\
\hline
\end{tabular}

\section{IV. - APPLICATIONS PRATIQUES}

A l'échelon industriel cette méthode a été appliquée dans deux beurreries.

Nous reproduisons les résultats obtenus dans l'une d'entre elles sur 25 échantillons de crème répartis en 4 séries. L'examen bactériologique des échantillons lors de ces essais était limité au dénombrement des germes totaux.

a) Résultats

\begin{tabular}{c|c|c|c|c|c|c}
\hline & Classement par le \\
test au \\
D.N.P.H.
\end{tabular}




\begin{tabular}{|c|c|c|c|c|c|c|}
\hline \multirow{11}{*}{ 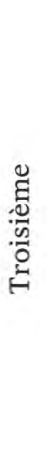 } & 1 & 89 & 2 & Acide, goût de vieille crème & $2^{\mathrm{me}}$ & II-III \\
\hline & 2 & 51 & 3 & Assez bonne, goût franc .. & $1^{\text {re }}$ & I \\
\hline & 3 & 68 & 6 & Un peu amère $\ldots \ldots \ldots c$ & $2^{\mathrm{me}}$ & II-III \\
\hline & 4 & 115 & 2 & Très acide, goût vieux, al- & $7 \mathrm{me}$ & TI \\
\hline & 5 & 75 & 6 & Acide, goût de vieux très & $2^{3,10}$ & $11-11$ \\
\hline & 6 & 61 & 5 & 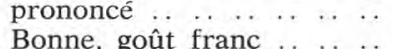 & $\begin{array}{l}2^{\mathrm{me}} \\
1^{\text {re }}\end{array}$ & $\begin{array}{l}\text { II-III } \\
\text { I }\end{array}$ \\
\hline & 7 & 120 & 2 & Très acide, goût de vieux & & \\
\hline & 8 & 47 & 3 & Goût légèrement malté, fran- & $2^{\text {me }}$ & II-III \\
\hline & & & & che dans l'ensemble $\ldots \ldots$ & $2^{\mathrm{me}}$ & II-III \\
\hline & 9 & 48 & 4 & Goût légèrement malté .. & $1^{\text {re }}$ & I \\
\hline & 10 & 50 & 3 & $\begin{array}{l}\text { Très épais, goût altéré, lé- } \\
\text { gèrement malté } \ldots \ldots \ldots \ldots\end{array}$ & & \\
\hline \multirow{5}{*}{ ్ٍ } & 1 & 85 & & Fermentée, acide $\ldots \ldots \ldots$ & $2^{\text {me }}$ & II-III \\
\hline & 2 & 45 & $\underbrace{\infty}_{0}$ & Très épaisse, un peu amère & $2^{\mathrm{me}}$ & II-III \\
\hline & 3 & 63 & 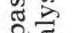 & Altérée $\ldots \ldots \ldots \ldots \ldots \ldots$ & $2^{\mathrm{me}}$ & II-III \\
\hline & 4 & 38 & 2 & $\begin{array}{llllllll}\text { Bonne } & \ldots & \ldots & \ldots & \ldots & \ldots & \ldots & \ldots\end{array}$ & $1^{\text {re }}$ & I \\
\hline & 5 & 25 & & Très liquide, franche ... .. & $1^{\text {re }}$ & I \\
\hline
\end{tabular}

\section{b) Interprétation}

En examinant le tableau ci-dessus, nous constatons qu'il est facile de distinguer les crèmes de très bonne qualité des moins bonnes. Par contre, il est plus difficile de faire une distinction très nette entre les échantillons de qualité moyenne qui donnent une coloration par le test au D.N.P.H. se situant dans la zone intermédiaire. Ces échantillons de crème représentent malheureusement le pourcentage le plus élevé des crèmes de ramassage. Cependant l'avis de tous les techniciens qui dans leur usine ont testé cette méthode est que les appréciations organoleptiques concordaient dans 80 p. 100 des cas avec les résultats du test au D.N.P.H.

\section{V. - CONCLUSION}

Les modalités de la technologie du beurre obtenu à partir de crèmes fermières, ne permettent pas de se baser sur une appréciation directe ou indirecte de l'acidité des crèmes pour établir un classement de celles-ci.

L'épreuve de sédimentation des moisissures n'est pas pratique et pas applicable à l'échelon industriel. La détermination des indices classiques d'altération des corps gras est longue et risque de faire appel à un équipement important.

Nous avons envisagé un test basé sur la réaction des composés aldéhydiques et cétoniques résultant de l'altération de la matière 
grasse de la crème, avec la 2-4 dinitrophénylhydrazine et la coloration par la potasse alcoolique des hydrazones formées. Les résultats obtenus au laboratoire d'une part, et à l'échelon industriel d'autre part semblent donner des résultats encourageants.

\section{S u m m a ry}

By this studies, we believe having shown the interest, for the industrial butter-man, to obtain from his suppliers, good-qualited creams predisposing to the preservation of the final producted-butter.

\section{Remerciements}

Nous remercions $M$. FRANÇOIS, ancien Directeur de la Laiterie Coopérative du Mans pour le concours qu'il a bien voulu nous accorder dans la réalisation de ce travail.

Nos remerciements s'adressent également à $M$. LABLANCHY, Directeur adjoint de la Coopérative d'Auxerre pour les appréciations qu'il nous a données, ainsi que pour les échantillons qu'il nous a gracieusement fournis lors de cette étude.

Nos remerciements s'adressent également à M. TAUPIN, Ingénieur E.N.S.A. (Grignon), pour nous avoir communiqué les résultats de ses observations en usine.

\section{Références bibliographiques}

[1] Alifax (R.) (1958). - Utilisation du monolaurate de sorbitol comme agent émulsionnant des graisses, dans les milieux destinés à la recherche des micro-organismes lipolytiques. Le Lait, $\mathrm{n}^{\circ}$ 373-374, p. 129-134.

[2] Wildman (1937). - Microscopic Method for estimating of Mild in cream. J. Assoc. of Agri. Chem, 20, p. 92-100.

Reçu pour publication en décembre 1969. 